\title{
Compact Printed C-shaped Monopole Antenna with Chip Inductor
}

\author{
Qi Luo/ H.M.Salgado \\ INESC Porto / FEUP, University of Porto \\ Porto, Portugal \\ qiluo@ieee.org / hsalgado@fe.up.pt
}

\author{
J.R.Pereira \\ IT Aveiro / University of Aveiro \\ Aveiro, Portugal \\ jrp@ua.pt
}

\begin{abstract}
This paper presents one compact printed monopole antenna with one chip inductor embedded on the radiation elements. By introducing the chip inductor, the resonant frequency of the original antenna can be decreased by more than $37 \%$ while there is no significant influence on its radiation characteristics including radiation efficiency and antenna gain. Meanwhile, after adding the chip inductor, the higher mode of the original antenna can also be brought down to a lower frequency, which makes the proposed antenna resonate at the dual frequency band and simultaneously have a compact size. This antenna has a $\mathrm{C}$-shaped geometry and its parameters including the location of the chip inductor are optimized by doing parametrical studies in Ansoft HFSS. The measurement and simulation results show that the proposed $\mathrm{C}$-shaped monopole antenna can operate at $2.55-2.65 \mathrm{GHz}$ and $5.1-5.3 \mathrm{GHz}$ with peak gain of 2.2 and $4.7 \mathrm{~dB}$, respectively. Moreover, according to the simulation results, the radiation efficiency of this antenna at both bands is around $90 \%$.
\end{abstract}

Keywords-printed monopole antenna; chip inductor; mutliband antenna

\section{INTRODUCTION}

Compact multiband antenna design has attracted much research interests in recent years due to the rapid development of wireless communications and growing needs of small size wireless devices. Different approaches, reported in the literature, have been used to the design of compact antennas. These methods include the use of folded spherical antennas [1], stacked antenna structure $[2,3]$ and antennas with high permittivity substrate [4], among others.

The planar monopole antenna is one of the attractive candidates for compact multiband antenna design because of its advantages such as low profile, easy fabrication and broad band operation characteristics. Instead of using conventional methods, which include using resonant elements of different shape or using multi-armed structures [5-7], several new approaches have been introduced. In [8], a triple band compact monopole antenna that can cover the dual band Wi-Fi and WiMAX band was achieved by using a single cell metamaterial loading. Moreover, introducing embedded capacitors or chip inductors into the design of printed monopole antennas has also been proposed. In [9], a small size printed multi-branch monopole antenna with a printed narrow strip as the distributed inductor has been proposed for GSM/UMTS mobile phone applications. Using the same concept, instead of using distributed inductors, chip inductors can be directly embedded into the antenna resonant element, which can result in even smaller size designs. By using this technique, two printed monopole antennas with similar radiation performance for mobile phone wireless wide area network (WWAN) applications have been designed $[10,11]$.

In this paper, the techniques proposed in $[10,11]$ is further applied to design one printed monopole antenna for dual band WLAN applications. The objective of this study is to design one compact printed monopole antenna that can resonant at 2.4 and $5.2 \mathrm{GHz}$ WLAN frequencies. The proposed monopole antenna has only one resonant element and the dual band operation is achieved by embedding one chip inductor of $20 \mathrm{nH}$ to bring down the resonant modes of the original antenna. Compared to the case that there is no inductor embedded, it is observed that with the existence of the chip inductor, the lowest resonant frequency of the antenna can be reduced by more than $37 \%$. The proposed antenna has a C-shaped structure and the size of this antenna is only $15 \times 8.6 \mathrm{~mm}^{2}$. Simulation results indicate that this proposed antenna has moderate gain and high radiation efficiency.

\section{ANTENNA DESIGN}

\section{A. Antenna Structure}

Fig. 1(a), (b) and (c) shows the top view, back view and side view of the proposed $\mathrm{C}$-shaped monopole antenna. The antenna element is printed on the top side of the substrate while the ground plane is located at the bottom side. Behind the radiation elements, there is no ground. The chip inductor, Coilcraft 0402HP series with inductance of $20 \mathrm{nH}$, is embedded on the top of the C-shape element (see L4 in Fig.1 (a)). The antenna occupies a space of $15 \times 8.6 \mathrm{~mm}^{2}$ and the total size of this antenna including the ground plane and feeding line is $15 \times 35 \mathrm{~mm}^{2}$. To achieve a better impedance match at both bands, the length and width of each branch of the antenna elements are optimized by doing numerical simulations in Ansoft HFSS, which is a 3D EM simulation software based on Finite Element Methods (FEM). This antenna is designed on substrate Roger 4003 with thickness of $0.8 \mathrm{~mm}$ and relative permittivity of 3.38 .

Qi Luo acknowledges the support for a scholarship from Fundação para a Ciência e Tecnologia. We also acknowledge funding from FCT and program POCTI/FEDER under the National Plan for Scientific Hardware Renewal with grant REEQ/1272/EEI/2005 


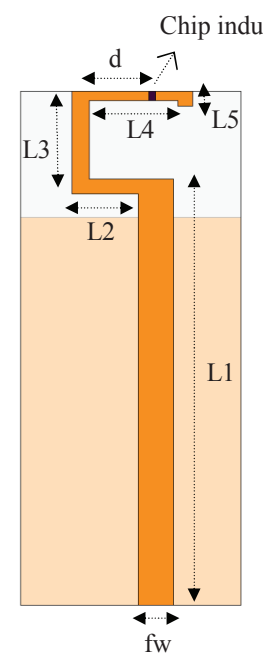

(a)

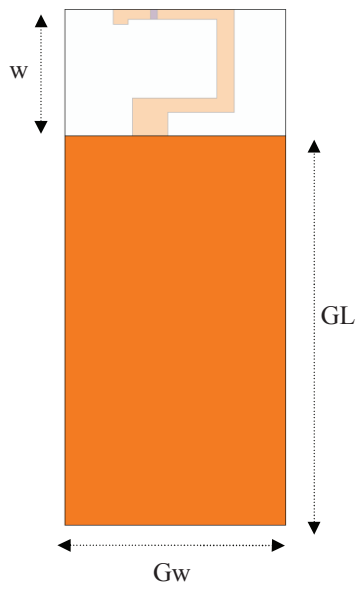

(b)

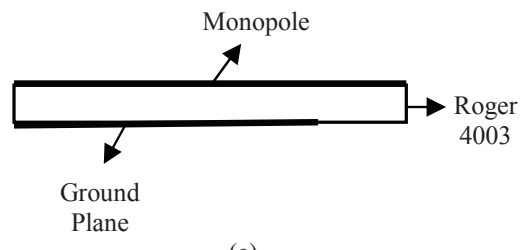

(c)

Figure 1 (a) Top view, (b) Back view and (c) side view of the proposed $\mathrm{C}$ shaped Monopole antenna with embedded chip inductor

The values of the parameters shown in Fig. 1 are given in table 1, where w2, w3, w4 and w5 represent the corresponding width of each microstrip branches.
Table 1: Parameters of the proposed antenna

\begin{tabular}{|c|c|}
\hline Parameter & Value (mm) \\
\hline GL & 26.4 \\
\hline Gw & 15 \\
\hline w & 8.6 \\
\hline fw & 2.4 \\
\hline $\mathrm{d}$ & 4 \\
\hline L1 & 28 \\
\hline L2/w2 & $4.5 / 1$ \\
\hline L3/w3 & $6 / 1.2$ \\
\hline L4/w4 & $7 / 0.65$ \\
\hline L5 /W5 & $0.35 / 1$ \\
\hline
\end{tabular}

\section{SIMULATION AND EXPERIMENTAL RESULTS}

\section{A. Return Loss}

Fig. 2 shows the comparison between the simulated and measured return loss (S11) of the proposed monopole antenna. The differences between the simulation and experiment results is mainly due to the accuracy of the simulation model and the errors introduced by soldering the chip inductor, which has a small size of only $1.12 \times 0.66 \mathrm{~mm}^{2}$, to the antenna element. During the simulation process, to simplify the simulation model, the inductor was simulated by assigning RLC boundary conditions to a rectangular of size $0.5 \times 0.65 \mathrm{~mm}^{2}$ in Ansoft HFSS, which is a simplified consideration. However, from both simulated and measured results, it can be observed that the proposed monopole antenna clearly exhibits two operational bands $(\mathrm{S} 11<-10 \mathrm{~dB})$ at 2 and $5 \mathrm{GHz}$ band. The experimental results show that the proposed antenna has a $-10 \mathrm{~dB}$ return loss over $2.55-2.65 \mathrm{GHz}$ and $5.1-5.3 \mathrm{GHz}$.

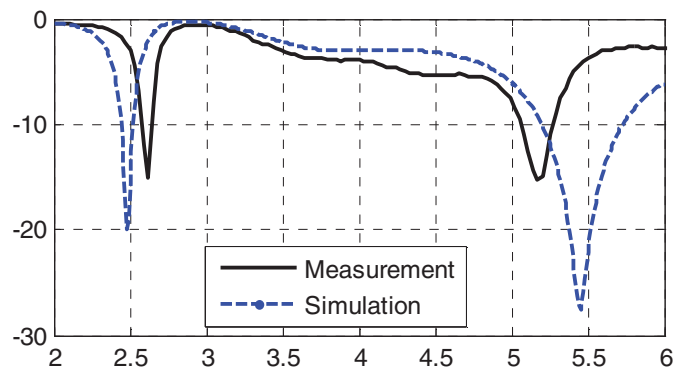

Figure 2: Comparison between the measured and simulated return loss

In order to show the effectiveness of embedding the chip inductor to reduce the size of one monopole antenna design, one more antenna prototype has been fabricated. This prototype has exactly the same parameters and dimensions as the 
proposed antenna except that there is no chip inductor embedded. Fig. 3 compares the return loss of the two antennas, the proposed antenna with chip inductor and the one without the chip inductor.

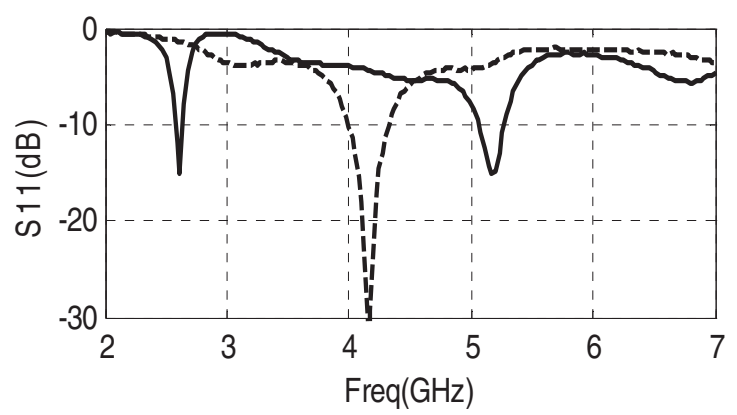

Figure 3: Comparison of the measured return loss between the proposed antenna with (solid line) and without (dash line) the chip inductor

From Fig.3, it can be seen that without the chip inductor, the monopole antenna resonates only at $4.15 \mathrm{GHz}$. After adding the chip inductor, this antenna can be made to resonant at $2.6 \mathrm{GHz}$, which is a frequency reduction of more than $37 \%$. Moreover, another resonant frequency at $5.2 \mathrm{GHz}$ is also observed. By proper adjustment of the relevant parameters of the antenna, it is possible to make this antenna operate at the desired frequencies for WLAN dual band application.

\section{B. Radiation Pattern}

Fig. 4 (a) and (b) shows the measured radiation patterns at both E-and H-plane of the proposed antenna at 2.55 and $5.2 \mathrm{GHz}$, respectively. At the higher band, this antenna radiates as a normal monopole antenna. However, at the lower band, the proposed antenna does not have an omnidirectional radiation pattern at its H-plane and at its E-plane, the main beam has been tilted by around 30 degrees.

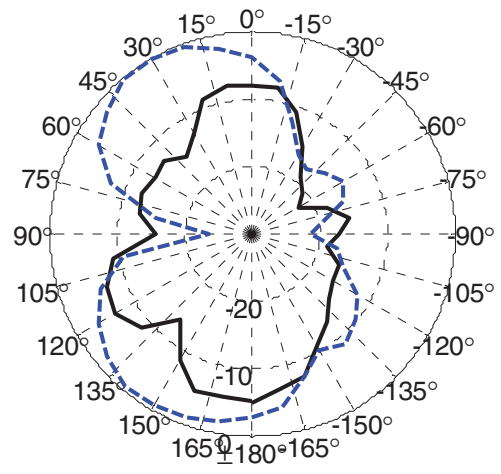

(a)

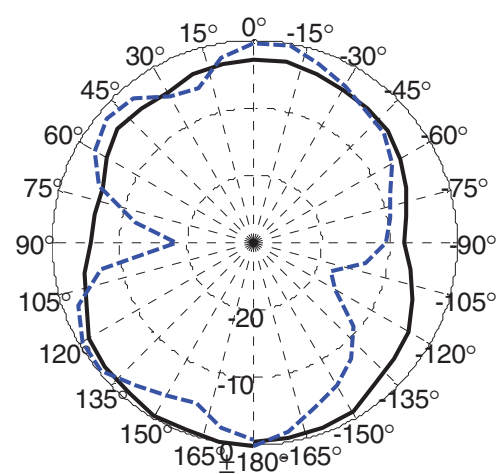

(b)

Figure 4: Measured radiation patter at H-plane (solid line) and E plane (dashed line) at (a) $2.55 \mathrm{GHz}$ and (b) $5.25 \mathrm{GHz}$

Fig. 5 (a) and (b) shows the simulated peak gain of this antenna at its lower and higher band, respectively. The simulation results show that the peak gain of the proposed antenna at $2.55 \mathrm{GHz}$ is $2.2 \mathrm{~dB}$ while the peak gain at $5.25 \mathrm{GHz}$ is $4.7 \mathrm{~dB}$. From the simulation results, it is also found that the radiation efficiency at both bands is around $90 \%$, which means that introducing the chip inductor does not greatly influence the radiation efficiency of the original antenna.

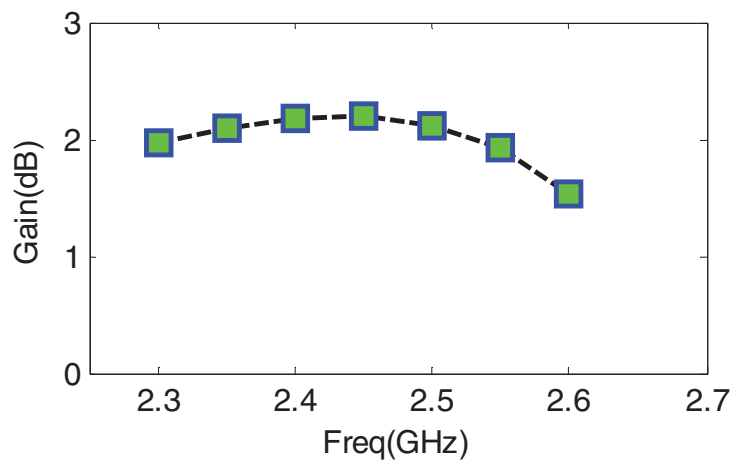

(a)

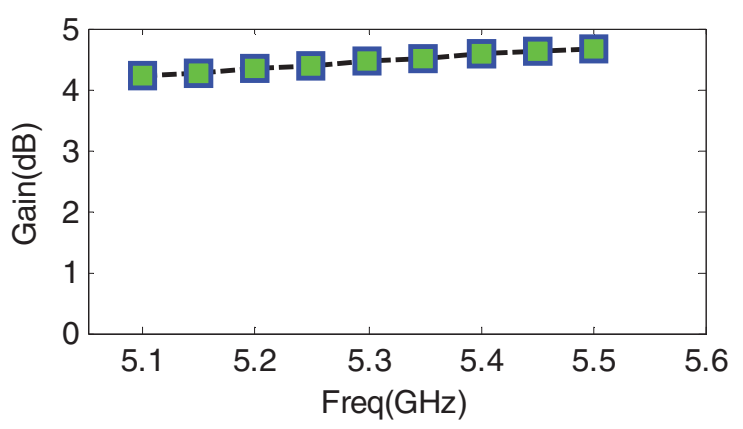

(b)

Figure 5: Simulated peak gain of the proposed antenna 


\section{CONCLUSIONS AND FUTURE STUDIES}

In this paper, one compact printed monopole antenna for WLAN dual band application has been presented. The compact size of this antenna is achieved by embedding one chip inductor on the antenna element. The simulation and experiment results show that by using this technique, the lower resonant frequency of the antenna can be reduced by more than $37 \%$ and the higher mode of the original antenna can also be decreased to a lower frequency band while there is no significant influence on the antenna's radiation efficiency.

The future work includes refinement of the simulation model of the antenna to reach a more accurate prediction of the antenna's performance and the measurement of the radiation efficiency using the Wheeler Cap methods $[12,13]$.

\section{REFERENCES}

[1] S.R.Best, 'The Radiation Property Of Electrically Small Folded Spherical Helix Antennas', IEEE Trans. Antennas Propag.,Vol.52, No.4, pp.953-960, April 2004

[2] A.A.Deshmukh and G.Kumar, 'Comapct Broadband Stacked Microstrip Antennas', IEEE Antennas and Propagation Society International Symposium 2006, Albuquerque, July 2006

[3] E.Tentzeris, R.L.Li and etc, 'Design Of Comapct Stacked-Patch Antennas On LTCC Technology For Wireless Communication Applications', IEEE Antennas and Propagation Society International Symposium 2002, August 2002
[4] A. Hoorfar and A. Perrotta, 'An Experimental Study of Microstrip Antennas on Very High Permittivity Ceramic Substrates and Very Small Ground Planes', IEEE Tran. Antennas Propag, Vol. 49, No. 4, April 2001

[5] S.R.Best,'On The Resonant Properties Of The Koch Fractal And Other Wire Monopole Antennas', IEEE Antennas and Wireless Propag.Letter, Vol.1, 2002

[6] Y.Song, Y.C.Jiao and etc, 'Compact Pritned Monopole Antenna For Multiband WLAN Applications', Microwave and Optical technonolgy letters, Vol.50, No.2, Februray, 2008

[7] Q.Luo,JR.Pereira and H.M.Salagdo, 'Fractal Monopole antenna for WLAN USB dongle', 2009 Loughborough Antenna \& Propogation Conference, November 2009

[8] J.Zhu,M.A.Antoniade and G.V.Eleftheriades, 'A Compact Tri-Band Monopole Antenna With Single Cell Metamaterial Loading', IEEE Trans. Antennas Propag.,Vol.58, No.4, pp.1031-1038, April 2010

[9] C.Chang and K.Wong, 'Small-Size Printed Monopole With A Printed Distributed Inductor for Pentaband WWAN Mobile Phone Application', Microwave and Optical Technology letters.,Vol.51, No.12, pp.29032908, December 2009

[10] K.Wong and S.Chen, 'Printed Single-Strip Monopole Using A Chip Inductor For Penta-Band WWAN Operation In The Mobile Phone', IEEE Trans. Antennas Propag.,Vol.58, No.3, pp.1011-1014, March 2010

[11] T. Kang and K.Wong, 'Chip-Inductor-Embedded Small-Size Printed Strip Monopole For WWAN Operation In The Mobile Phone', Microwave and Optical Technology letters.,Vol.51, No.4, pp.996-971, April 2009

[12] D.M.Pozar, 'Comparison Of Three Methdods For The Measurement Of Printed Antenna Efficiency', IEEE Trans. Antennas Propag.,Vol.36, No.1, pp.136-139, Jan 1988

[13] H.Choo, R.Rogers and H.Ling, 'On The Wheeler Cap Measurement Of The Efficency Of Microstrip Antennas', IEEE Trans. Antennas Propag.,Vol.53, No.7, pp.2328-2332, July 2005 